\title{
TITLE:
}

\section{Large and small Fermi-surface spin density waves in the Kondo lattice model}

\author{
$\operatorname{AUTHOR}(S)$ :
}

Peters, Robert; Kawakami, Norio

\section{CITATION:}

Peters, Robert ...[et al]. Large and small Fermi-surface spin density waves in the Kondo lattice model. Physical Review B 2015, 92(7): 075103.

ISSUE DATE:

2015-08-03

URL:

http://hdl.handle.net/2433/202047

RIGHT:

(C2015 American Physical Society 


\title{
Large and small Fermi-surface spin density waves in the Kondo lattice model
}

\author{
Robert Peters ${ }^{1, *}$ and Norio Kawakami ${ }^{2}$ \\ ${ }^{1}$ Computational Condensed Matter Physics Laboratory, RIKEN, Wako, Saitama 351-0198, Japan \\ ${ }^{2}$ Department of Physics, Kyoto University, Kyoto 606-8502, Japan
}

(Received 27 April 2015; published 3 August 2015)

\begin{abstract}
We demonstrate the existence of metallic spin density waves (SDWs) in the Kondo lattice model on a square lattice for a wide range of parameters by means of real space dynamical mean-field theory. In these SDWs, the spin polarization as well as the charge density depend on the lattice site and are modulated along one direction of the square lattice. We show that within this phase of metallic SDWs the Fermi surface changes from small to large, when the coupling strength is increased. Furthermore, the transition between the large Fermi-surface SDW phase and the paramagnetic phase is of second order, while the transition between the small Fermi-surface SDW phase and the paramagnetic phase is of first order. A local quantum critical point is thus avoided in our calculations by undergoing a first-order phase transition.
\end{abstract}

DOI: 10.1103/PhysRevB.92.075103

PACS number(s): 71.10.Fd, 71.27.+a, 75.30.Fv

\section{INTRODUCTION}

Strongly correlated materials are of special interest in condensed matter physics because they often exhibit remarkable phenomena which cannot be explained by single-particle theory. A particular class of strongly correlated materials are heavy fermion systems, in which quantum criticality accompanied by non-Fermi liquid behavior or unconventional superconductivity can often be observed [1-11]. Heavy fermion materials include strongly interacting $f$ electrons, which are hybridized with conduction spd bands. The main driving forces in these materials are the Kondo screening, which favors a paramagnetic state, and the Ruderman-Kasuya-KittelYosida (RKKY) interaction, which favors a magnetically long-range ordered state. Especially, when both effects are of comparable strength, fascinating many-particle phenomena such as quantum criticality and non-Fermi-liquid physics can arise.

It has been found that quantum phase transitions in heavy fermion systems can be categorized into two classes: (i) quantum fluctuations at the phase transition arise solely due to the magnetic order and the Fermi surface behaves smoothly across the transition, which corresponds to the Hertz-MillisMoriya type of quantum critical point (QCP) [12-14] and (ii) exactly at the quantum phase transition between magnetism and paramagnetism, not only the magnetic order vanishes, but also the Kondo screening vanishes and the Fermi surface changes abruptly, which is referred to as local quantum criticality $[1,15]$. While $\mathrm{CeNi}_{2} \mathrm{Ge}_{2}$ is an example for the HertzMillis-Moriya type of QCP [6], there is evidence that the QCP observed in $\mathrm{YbRh}_{2} \mathrm{Si}_{2}$ corresponds to a local quantum critical point [16-21]. These experimental and theoretical results on quantum phase transitions in heavy fermion systems have been summarized in the global phase diagram [2,8-10,22-27], which includes small/large Fermi-surface antiferromagnetic phases and small/large Fermi-surface paramagnetic phases.

A commonly used theoretical model to describe heavy fermion systems is the Kondo lattice model, in which effective local moments originating from the interacting $f$ electrons

*robert.peters@ @iken.jp are coupled to conduction electrons. The Kondo lattice model reads [28-32]

$$
\begin{aligned}
H & =t \sum_{\langle i, j\rangle \sigma} c_{i \sigma}^{\dagger} c_{j \sigma}+J \sum_{i} \vec{S}_{i} \vec{s}_{i}, \\
\vec{s}_{i} & =c_{i \sigma_{m}}^{\dagger} \vec{\rho}_{\sigma_{m} \sigma_{n}} c_{i \sigma_{n}},
\end{aligned}
$$

where $c_{i \sigma}^{\dagger}$ creates an electron with spin direction $\sigma$ on lattice site $i$. The first term in Eq. (1) describes the hopping of conduction electrons, and the second term describes the coupling of the conduction electrons to the localized spins with interaction strength $J$. Throughout this paper, we assume an antiferromagnetic coupling, $J>0$, and take the hopping constant $t$ as unit of energy. All calculations are performed on a two-dimensional square lattice.

In this paper, we examine magnetism and quantum phase transitions in the Kondo lattice model by means of the dynamical mean-field theory (DMFT) [33-35], which has been extensively used for this purpose [36-58]. Although these previous studies include calculations for the antiferromagnetic state and the magnetic quantum phase transition, they were performed for the Néel state with commensurate ordering vector. Exactly at half-filling for a particle-hole symmetric lattice, the Néel state is insulating. Thus the behavior of the Fermi surface across the quantum critical point cannot be studied. Some of these previous studies also used the Néel state away from half-filling within the metallic antiferromagnetic phase. However, away from half-filling, one can expect that the Néel state becomes energetically unstable towards incommensurate magnetic states, as has also been found in many experimental systems by neutron scattering, e.g., for $\mathrm{CeRu}_{2} \mathrm{Si}_{2}$ [59], $\mathrm{CeCu}_{2} \mathrm{Si}_{2}$ [60], $\mathrm{CeRhIn}_{5}$ [61], etc. A theoretical study using classical spins already proved the existence of incommensurate SDWs [62]. However, due to the approximation of classical spins, many-particle effects, particularly the Kondo effect, are absent in Ref. [62].

In this paper, we use the DMFT, and explicitly include the possibility of incommensurate magnetic states in the Kondo lattice model by using large-scale real-space DMFT (RDMFT). We will focus on the analysis of metallic SDW states away from half-filling, which have not been carefully 
studied yet, and analyze static and dynamical properties with particular emphasis on the behavior of the Fermi surface across the quantum phase transition.

This paper is organized as follows. In the next section (Sec. II), we will shortly explain the methods used in this paper. This is followed by the section (Sec. III), in which we will summarize the calculated phase diagram and explain the different types of SDWs observed in our calculations. Static (Sec. IV), dynamical properties (Sec. V), and the phase transitions between different phases (Sec. VI) are explained in more detail in the subsequent sections, before concluding the paper.

\section{METHOD}

We use large-scale real-space dynamical mean-field theory (RDMFT) to study the Kondo lattice model, Eq. (1), defined on a two-dimensional square lattice. In RDMFT, each lattice site of a finite cluster is mapped onto its own impurity model. This mapping is done by calculating the local Green's function of each lattice site via

$$
\mathbf{G}_{l o c}(z)=\int d k_{x} d k_{y}\left[z \cdot \mathbb{I}-\mathbf{H}_{k_{x}, k_{y}}-\Sigma\right]^{-1},
$$

where $\mathbf{H}_{k_{x}, k_{y}}$ is the hopping Hamiltonian of the chosen cluster and $\Sigma$ is the self-energy matrix. The momentum dependence of $\mathbf{H}_{k_{x}, k_{y}}$ arises thereby through the boundary conditions. For this study, we use $(20 \times 20),(60 \times 10)$, and $(30 \times 30)$ clusters with periodic boundary conditions. The selfenergy is diagonal and momentum-independent within this approximation; correlation effects between different lattice sites are not included. However, the self-energy depends on the lattice site of the cluster, which makes it possible to describe SDW states. From the local Green's function, a lattice-site-dependent hybridization is calculated via

$$
\Delta_{i i}(z)=z-\frac{1}{G_{l o c, i i}(z)}-\Sigma_{i i}(z),
$$

which determines the input for a single impurity Kondo model. These equations are reduced to the usual single-site DMFT, if the cluster consists of a single site. To obtain the self-energy of the Kondo impurity model, we use the numerical renormalization group (NRG) [63,64], which can calculate reliable self-energies at zero and finite temperature $[65,66]$. We then iterate Eqs. (2) and (3) until the self-energy matrix becomes self-consistent. In order to improve the stability of the iterative calculations, we do not directly use the self-energy as calculated by the NRG, but we add $50 \%$ of the self-energy of the last iteration, $\Sigma_{i+1}=0.5 \Sigma_{i}+0.5 \Sigma_{i-1}$, which is usually called "mixing" and frequently used in DMFT calculations. Recently, we have used similar calculations to study spin density and charge density waves in the Hubbard model [67].

\section{PHASE DIAGRAM}

Figure 1 summarizes our obtained results. Close to halffilling, the physics of the Kondo lattice model is dominated by the interplay of the Kondo effect and the RKKY interaction, as described by the Doniach phase diagram [28]. At weak coupling, where the RKKY interaction dominates, we observe

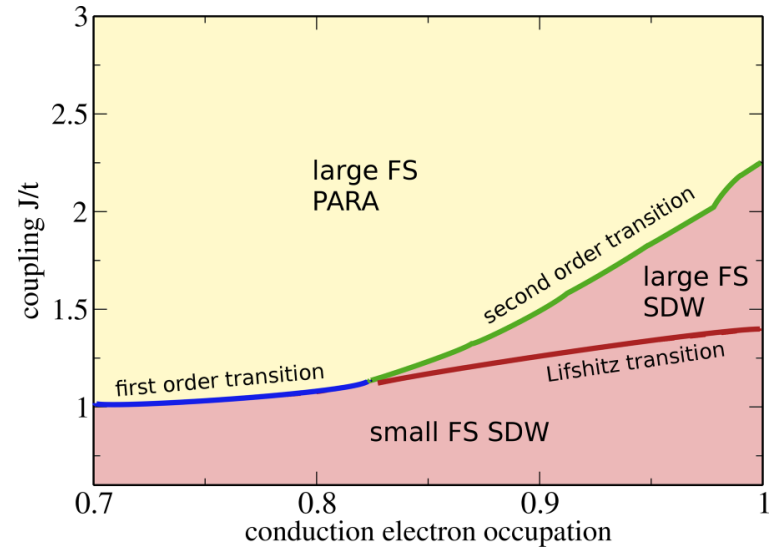

FIG. 1. (Color online) Phase diagram of the Kondo lattice model as calculated by RDMFT. The SDW phase away from half-filling is separated into a large Fermi-surface (FS) phase at strong coupling and a small Fermi-surface phase at weak coupling. A detailed explanation is given in the main text.

a magnetically ordered phase. At strong coupling, where the Kondo effect becomes dominating, a paramagnetic state is stabilized.

Exactly at half-filling, the model exhibits perfect nesting and we observe the well-known transition from an antiferromagnetic Néel state at weak coupling to a paramagnetic Kondo insulator at strong coupling. This continuous transition occurs approximately at $J_{c} / t \approx 2.2$ within DMFT for the square lattice. Notice that the Kondo lattice model on a square lattice remains insulating at half-filling for all interaction strengths, $J>0$, although the origin of the insulating gap gradually changes. Our obtained critical interaction strength for the transition between the Néel state and the Kondo insulator reasonably agrees with previous DMFT calculations [58]. We can thus reproduce previous results with our RDMFT calculations.

Away from half-filling, the homogeneous Néel state becomes unstable and changes into a phase of SDWs, in which the polarization of the conduction electrons as well as the localized spins are lattice-site dependent. For coupling strengths larger than the hopping amplitude, this SDW phase becomes unstable upon doping towards the large Fermi-surface paramagnetic state. On the other hand, for weak coupling, $J<t$, SDWs can be observed for any conduction band filling.

Even within this SDW phase, the modulation of the electron polarization and electron density depends on the model parameters. In Fig. 2, we compile information about typical SDWs, which can be observed in the vicinity of half-filling. For each type of SDW, (a)-(c), we show in the upper part of each panel false-color plots of the electron density (left plot) and the electron polarization (right plot) for a cluster of lattice sites. The lower plots of each panel show intersections of the above false-color plots.

Figure 2(a) shows the antiferromagnetic Néel state exactly at half-filling. In the Néel state, the polarization of the electrons changes its sign between nearest neighbors. The electron density, on the other hand, is unity for all lattice sites. Increasing the interaction strength beyond $J / t=2.2$, the antiferromagnetic state changes into the Kondo insulator 
(a)
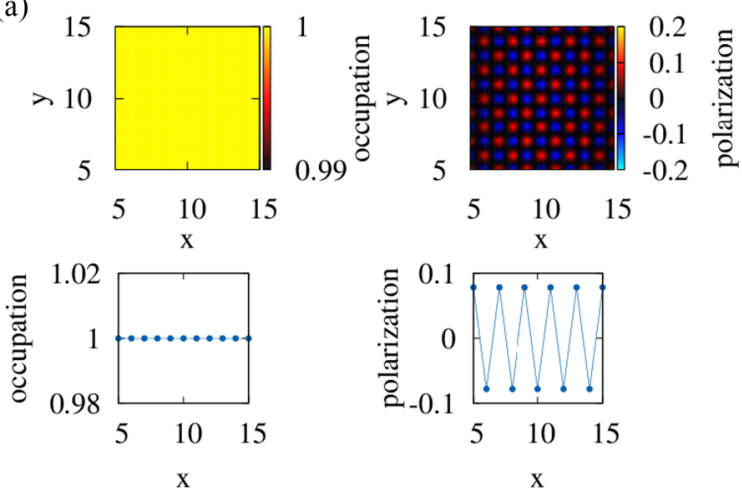

(b)
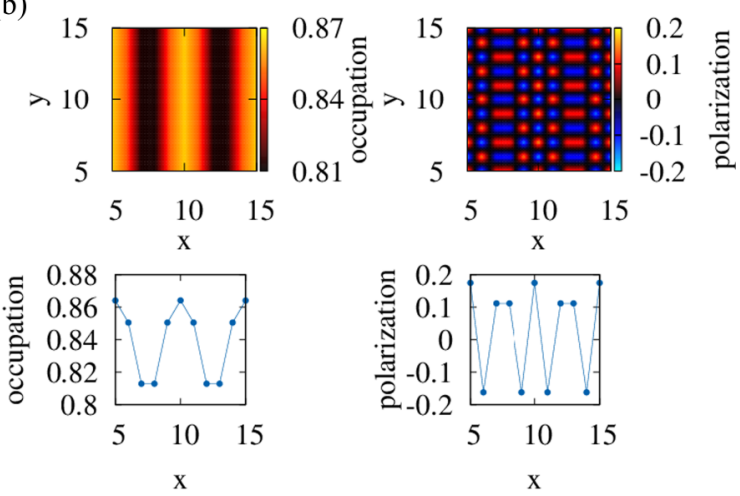

(c)
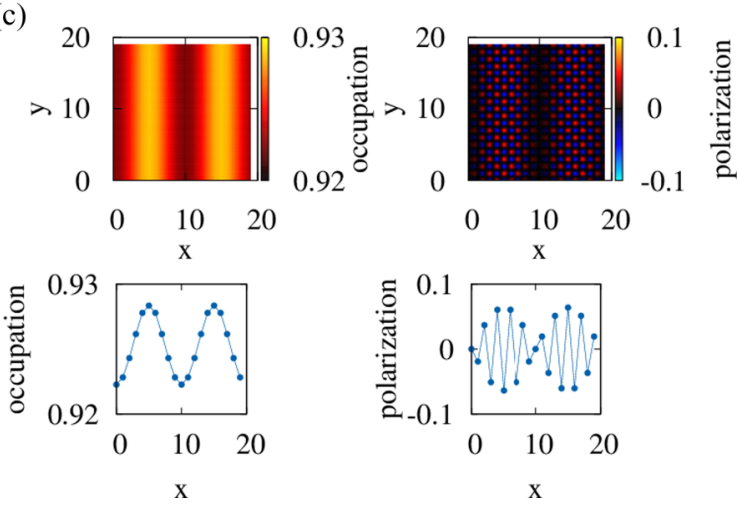

FIG. 2. (Color online) Different magnetic states close to halffilling: (a) antiferromagnetic Néel state exactly at half-filling $(J=2 t)$. (b) Doped SDW/CDW at weak interaction strength $(J=0.8 t)$. (c) Doped SDW/CDW close to the quantum critical phase transition $(J=1.8 t)$. For each state (a)-(c), we show a false-color-plot of the charge density (top left), a false-color-plot of the electron polarization (top right), an intersection of the charge-density plot for a given $y$ site (bottom left), and an intersection of the electron-polarization plot for a given $y$ site (bottom right).

(not shown in Fig. 2), where all lattices sites are occupied in average with a single electron and the polarization vanishes.

Away from half-filling, the perfect nesting, which exists exactly at half-filling, is lost, and the system can gain energy by modulating the electron polarization over the lattice sites. We have tried in our calculations different types of modulations, such as vertical SDWs and diagonal SDWs. It turned out that the most stable type of SDW on the square lattice is the vertical SDW, as shown in panels (b) and (c) of Fig. 2. The amplitude of the electron polarization is modulated along one direction of the square lattice. Along the other direction, the polarization shows a Néel-state-like "AB" oscillation. However, not only the polarization is modulated, but also the electron density depends on the lattice site: there are stripes of high electron density and stripes of low electron density. CDWs are well known in strongly correlated materials, e.g., high- $T_{c}$ cuprates, and have been detected there by x-ray scattering [68]. Within the regions of high electron density, we generally observe a Néel-state-like behavior; the polarization changes its sign between nearest neighbors. The behavior in the low-density regions, however, depends on the interaction parameter and the average occupation of the conduction electrons. For large interaction strengths close to the quantum critical transition, we observe that the polarization vanishes in the low-electrondensity regions, see Fig. 2(c). The Kondo effect becomes strong in these regions so that the localized moments are screened by the Kondo effect instead of forming a magnetically ordered state. On the other hand, for weak interaction strength, $J<t$, and especially for an average electron filling $n<0.9$, we observe that the low-electron-density regions are still magnetically polarized. We find that nearest-neighbor sites form ferromagnetic bonds along one axis, see Fig. 2(b). For weak interaction strengths, the localized moments cannot be screened by the Kondo effect, which is exponentially weak. Here the magnetic state is completely determined by the RKKY interaction. The localized moments are nearly fully polarized and form also inside the low electron-density region a magnetic state. Furthermore, the modulation of the polarization cannot be described anymore by a simple sine wave, but a more complex polarization pattern is realized. Due to a changed Fermi vector in this region, the system can gain energy by combining ferromagnetic bonds with antiferromagnetic ones. Experimentally, similar combinations of antiferromagnetic and ferromagnetic correlations have been observed in $\mathrm{YbRh}_{2} \mathrm{Si}_{2}[69,70]$.

\section{STATIC PROPERTIES OF THE SDW PHASE}

An important point, which was neglected in previous studies is that both the spin polarization and the electron density are modulated away from half-filling. In Fig. 3, we show the amplitude of the SDW (top) and the amplitude of the CDW (bottom) for different coupling strengths and conduction band fillings. We observe that the electron polarization within the SDW is largest for approximately $J \approx t$ exactly at half-filling. The spin polarization becomes weaker when doping the system away from half-filling. The amplitude of the CDW, however, is strongest for $J \approx t$ and conduction band fillings $\langle n\rangle \approx 0.9$. Exactly at half-filling, the electron density is homogeneous. Furthermore, the CDW is coupled to the SDW; within the paramagnetic state, no charge-density modulation exists. The Kondo effect alone is not sufficient to stabilize a CDW state for these conduction band fillings. With increasing coupling strength, the CDW vanishes faster than the SDW. However, this might be related to the fact that the SDW state can only be found very close to half-filling for strong interaction. In this parameter region, the ground state becomes more and more homogeneous, and the amplitude of the CDW is very small. 

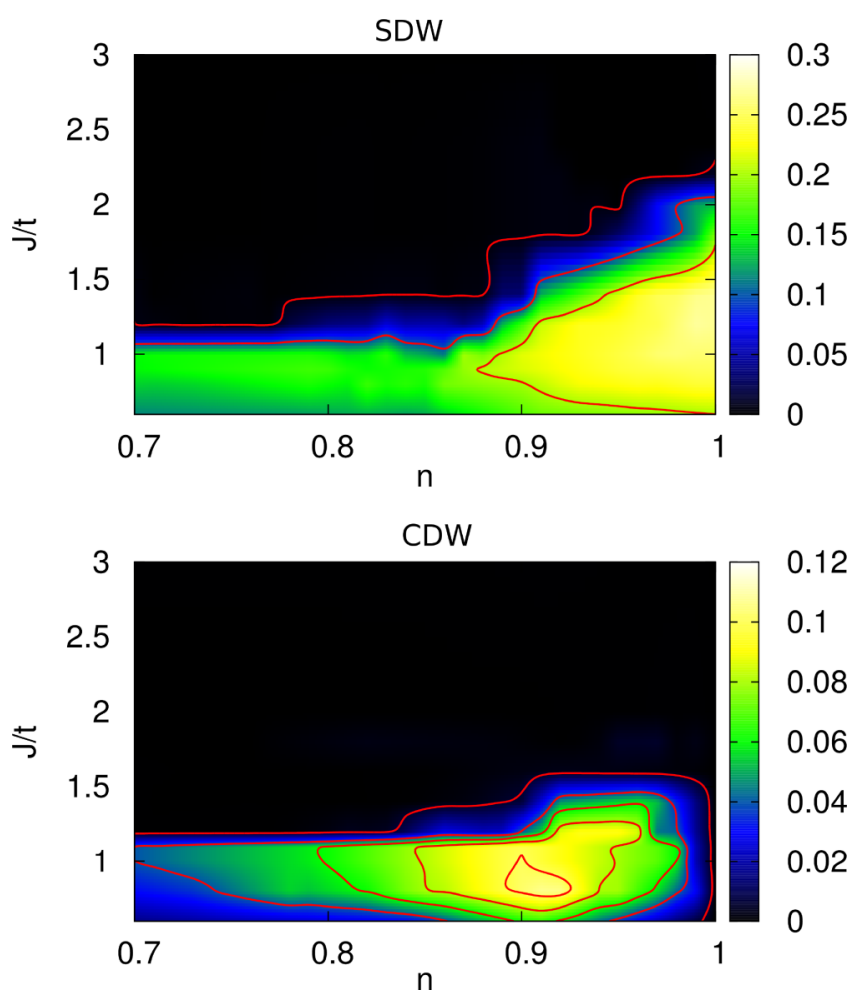

FIG. 3. (Color online) (Top) Electron polarization in the highdensity regions (corresponding to the maximal polarized electrons) for different coupling strengths and conduction band fillings. The red lines correspond to contour lines at $\left|n_{\uparrow}-n_{\downarrow}\right|=(0 ; 0.1 ; 0.2)$. (Bottom) The difference of the electron density between high- and low-density regions. The red lines correspond to contour lines at $\left|n_{\text {high }}-n_{\text {low }}\right|=(0 ; 0.03 ; 0.06 ; 0.09 ; 0.12)$.

The observed modulations of the spin polarization and the electron density are not independent of model parameters. In order to obtain information about the wavelength of the modulations, we have performed a Fourier transformation of the obtained DMFT solutions. For this purpose, we have used $(60 \times 10)$ clusters for our calculations. The Fourier transformation for the occupation and the polarization read

$$
\begin{aligned}
& \tilde{n}\left(k_{x}, k_{y}\right)=\frac{1}{N} \sum_{x, y} e^{i k_{x} x+i k_{y} y}\left(n_{\uparrow(x, y)}+n_{\downarrow(x, y)}-n_{\text {aver }}\right), \\
& \tilde{m}\left(k_{x}, k_{y}\right)=\frac{1}{N} \sum_{x, y} e^{i k_{x} x+i k_{y} y}\left(n_{\uparrow(x, y)}-n_{\downarrow(x, y)}\right),
\end{aligned}
$$

where we have subtracted the average filling of the lattice, $n_{\text {aver }}=\frac{1}{N} \sum_{x, y}\left(n_{\uparrow(x, y)}+n_{\downarrow(x, y)}\right)$, and $N$ corresponds to the number of lattice sites. The absolute values of these Fourier modes show distinct peaks at certain momenta in the Brillouin zone. We show these momenta in Fig. 4 for $J / t=0.8$. For this interaction strength, the SDW phase exists until close to quarter filling, where it changes into another magnetic phase via a first-order transition. The wavelength does not depend on the coupling strength $J$ within the weak coupling region of the phase diagram. If we neglect the parameter region of small hole doping, where the wavelength of the SDW exceeds the cluster size, the largest Fourier component in the SDW

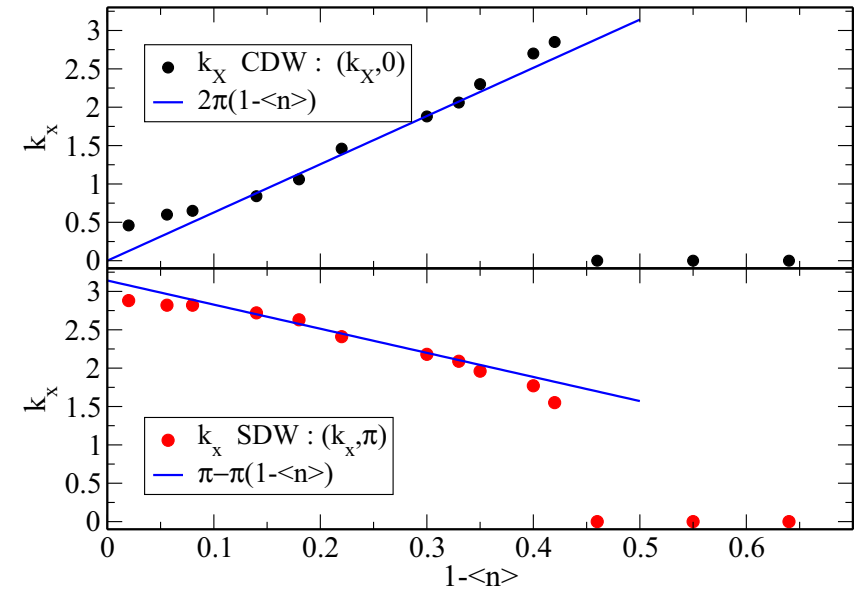

FIG. 4. (Color online) Strongest Fourier mode of the charge density (top) and spin polarization (bottom) for different conduction band fillings. The interaction value is $J / t=0.8$, for which the SDW phase exists until $\langle n\rangle \approx 0.6$.

and the CDW can be fitted by a linear function in the number of holes. Exactly at half-filling, we find a Néel state with modulation $\vec{k}=(\pi, \pi)$ without a CDW. Away from half-filling, this Néel state changes into an SDW with modulation $\vec{k}=$ $\pi(1-\langle h\rangle, 1)$ accompanied by a CDW with modulation $\vec{k}=$ $\pi(2\langle h\rangle, 0)$, where $\langle h\rangle=1-\langle n\rangle$ corresponds to the number of holes in the lattice. For stronger interaction values, the SDW phase vanishes upon doping into the paramagnetic heavy fermion state for small hole doping. The wavelength in this parameter region is too long, hence, we could not accurately analyze the doping dependence of it.

\section{SPECTRAL FUNCTION AND FERMI SURFACE}

As shown in Fig. 1, we have divided the SDW phase into two regions; one with large and one with small Fermi surface. We next want to show momentum-resolved spectral functions and the corresponding Fermi surfaces, which justify this distinction.

Figure 5 shows spectral functions for the paramagnetic phase (left), the strong-coupling SDW phase (middle), and the weak-coupling SDW phase (right). The lower panels correspond to magnifications around the Fermi energy of the upper panels. The green dashed line marks the Fermi energy. In the weak-coupling SDW phase (right panel in Fig. 5), we only find slight modifications from the noninteracting energy momentum dispersion. These modifications mainly occur close to the Fermi energy around $(\pi, 0),(0, \pi)$, and $(\pi / 2, \pi / 2)$, where we observe a suppression of the spectral weight. Compared to the noninteracting spectrum, additional weak bands occur around $(0,0)$ and $(\pi, \pi)$ below the Fermi energy. Especially the band that is close to the Fermi energy at $(\pi, \pi)$ is a remnant of the heavy fermion band, which, however, lies below the Fermi energy. The main contribution to the spectral weight at the Fermi energy originates from the noninteracting dispersion at $(\pi, 0),(0, \pi)$, and $(\pi / 2, \pi / 2)$.

The spectral functions of the paramagnetic metallic phase (left panel in Fig. 5) and the strong-coupling SDW phase 

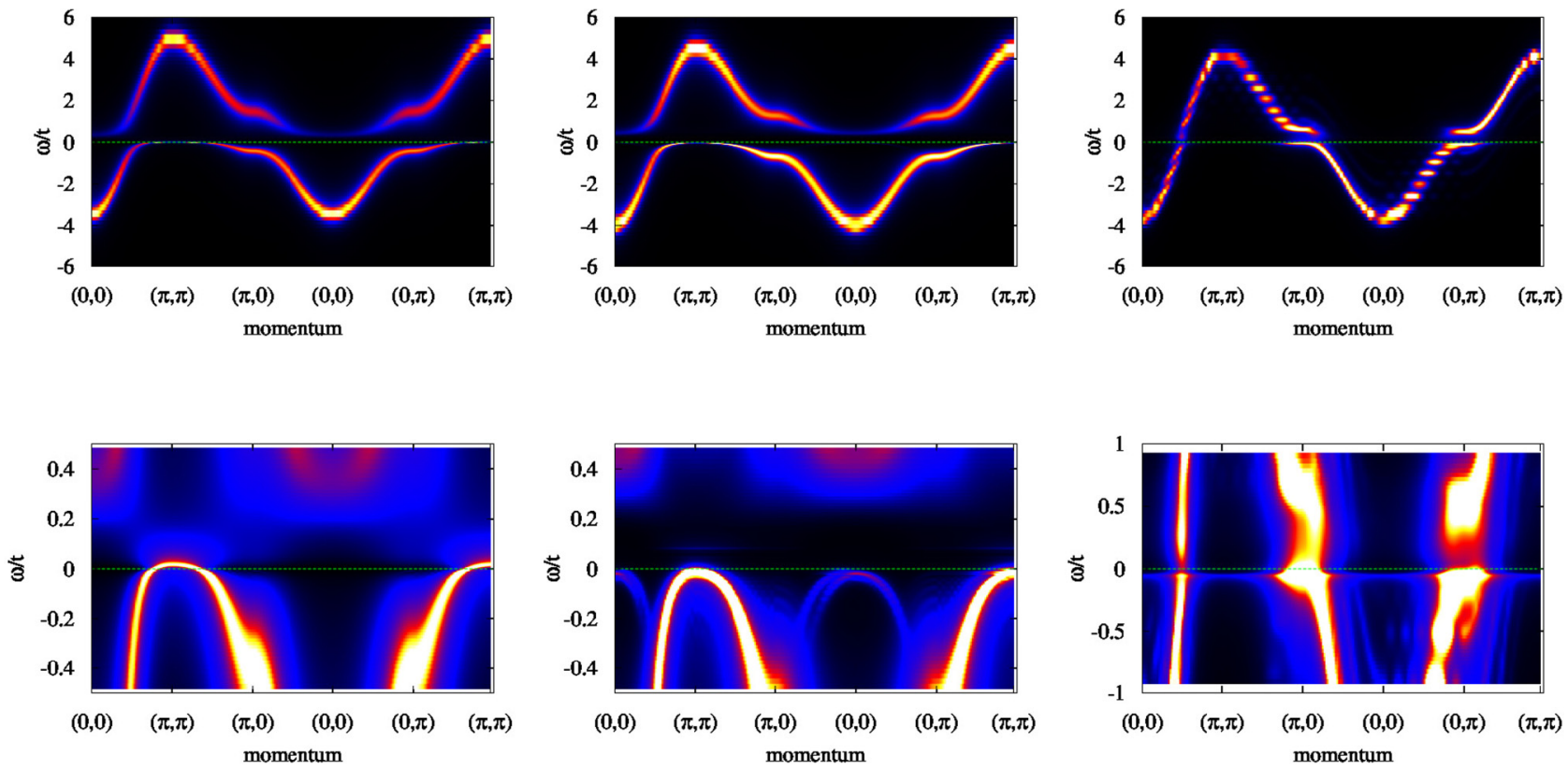

FIG. 5. (Color online) Spectral functions for the paramagnetic state (left), the strong-coupling SDW (middle), and the weak-coupling SDW state (right). The lower panel is a magnification of the upper panel around the Fermi energy.

(middle panel), differ substantially from the weak-coupling case. The bands that cross the Fermi energy in the non- and weakly-interacting case at $(\pi, 0)$ and $(0, \pi)$, are absent at the Fermi energy in this phase. While in the weakly interacting SDW phase there is a band that connects the low-energy parts $(\omega / t=-4)$ with the high-energy parts $(\omega / t=4)$, in the paramagnetic and the strong-coupling SDW state, there is a gap above the Fermi energy separating the low- and the high-energy parts of the spectrum. The noninteracting bands, which approach the Fermi energy at $(\pi, 0),(0, \pi)$, and $(\pi / 2, \pi / 2)$ are bent towards each other and cross the Fermi energy close to $(\pi, \pi)$. The spectrum of the paramagnetic state and the strong-coupling SDW state look thereby very similar to each other. The gap above the Fermi energy seems to be more pronounced in the SDW state. However, this is parameter dependent. Another general feature in the dispersion of the SDW state is that there is a band at $(0,0)$ close to the Fermi energy. This is a shadow band originating from the ordered state $[67,71]$.

From the spectral functions shown in Fig. 5, we can see that the Fermi surface changes within the SDW phase. For a better understanding, we have compiled the calculated Fermi surfaces for a few parameters in Fig. 6. Different rows show different transitions, which can be observed in the Kondo lattice model. The upper panels show the transition between the weak-coupling SDW phase and the paramagnetic phase at $\langle n\rangle \approx 0.8$ for $J / t=(0.6 ; 0.8 ; 1.0 ; 1.2 ; 1.4)$ (left to right). One can clearly observe a change in the Fermi surface topology at $J / t=1$. While in the left two panels the Fermi surface is small including only conduction electrons, the Fermi surface in the right two panels is large, which includes conduction electrons and localized moments. A similar change in the Fermi surface topology can be observed when increasing the interaction strength within the SDW phase (middle panels of Fig. 6). The Fermi surface clearly changes in the second panel from right for $J / t=1.2$ and $\langle n\rangle \approx 0.9$. Finally, the lower panels show the transition between the strong-coupling SDW and the paramagnetic phase for different conduction band fillings and $J / t=1.8$. The magnetic order vanishes in the middle panel for $\langle n\rangle \approx 0.93$. However, there is no abrupt change in the Fermi surface for strong coupling when the magnetic order vanishes. Summarizing these results, we find that the SDW phase away from half-filling is divided into a phase with small Fermi surface at weak coupling and a phase with large Fermi surface at strong coupling. We thus find a Lifshitz transition within the SDW phase, where the Fermi surface topology changes.

Although the Fermi surface vanishes exactly at half-filling due to the insulating nature of the system, a similar change in the energy-momentum dispersion can be observed. At weak coupling, the noninteracting bands approaching the Fermi energy at $(\pi, 0),(0, \pi)$, and $(\pi / 2, \pi / 2)$ are gapped out at the Fermi energy. Bands at $(\pi, \pi)$ and $(0,0)$ do not exist or are very weak. At approximately $J / t \approx 1.4$, this dispersion changes. The bands approaching the Fermi energy are bent towards each other. The spectral weight at $(\pi, 0)$ and $(0, \pi)$ close to the Fermi energy is shifted to $(\pi, \pi)$. Although none of these bands crosses the Fermi energy, we nevertheless observe a similar change in the energy-momentum dispersion at half-filling as for the doped system.

\section{PHASE TRANSITIONS}

As we have shown above, the Fermi surface changes within the SDW phase. We will show now that the phase transition between the SDW phase and the paramagnetic phase is also influenced by this change inside the SDW phase. In Fig. 7, we analyze the transition between the SDW state and the paramagnetic state at weak coupling and conduction-band filling $\langle n\rangle \approx 0.8$. This represents an example for the transition between the small Fermi-surface SDW and the large Fermisurface paramagnetic state. We show in Fig. 7 the polarization 

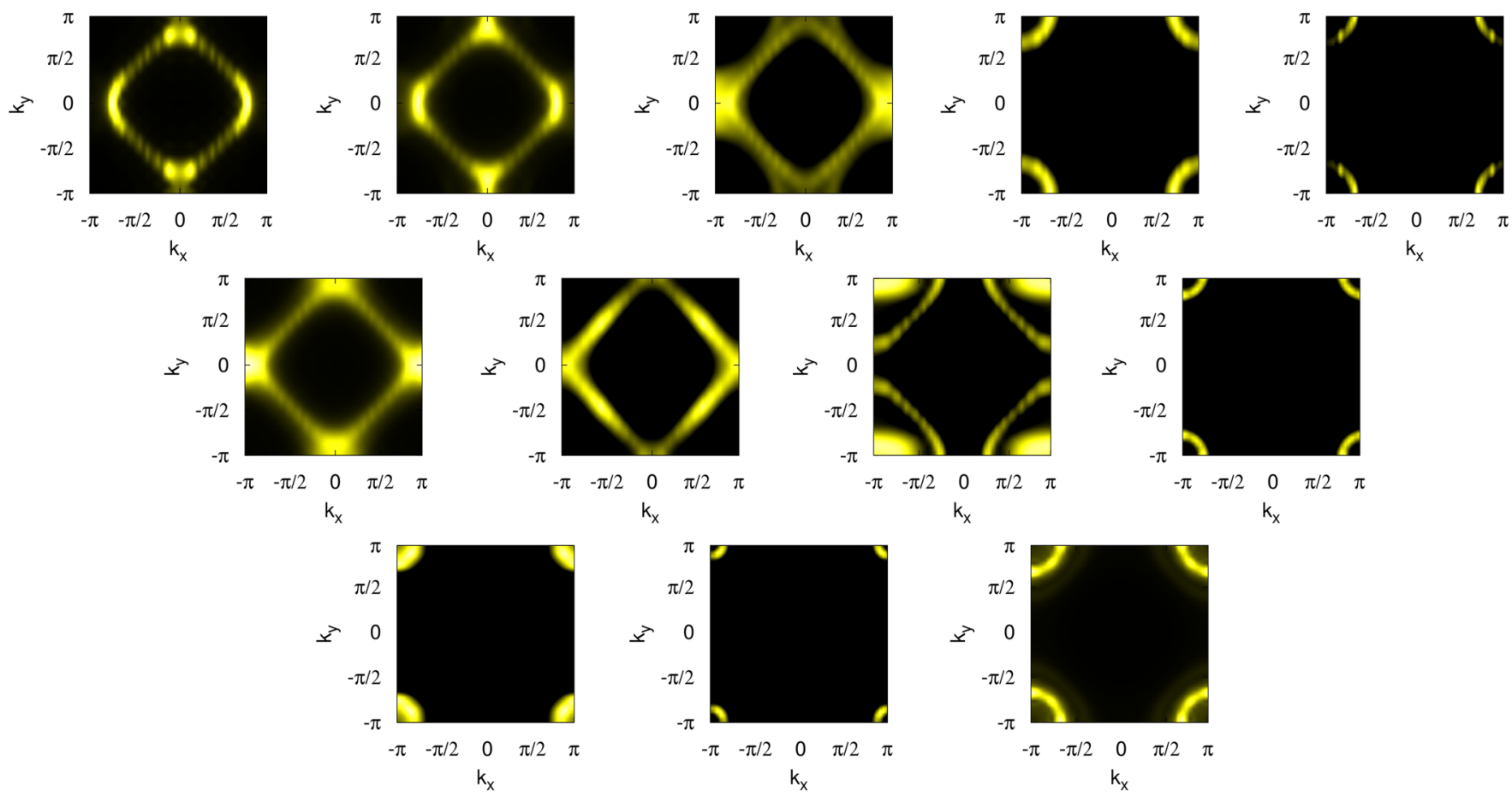

FIG. 6. (Color online) Calculated Fermi surfaces. (Top) Fermi surfaces across the transition between the weak-coupling SDW and the paramagnetic state at conduction band filling $\langle n\rangle \approx 0.8$ and (from left to right) $J / t=(0.6 ; 0.8 ; 1.0 ; 1.2 ; 1.4)$. The transition takes place at $J=t$. (Middle) Fermi surfaces inside the SDW phase for (from left to right) $J / t=(0.8 ; 1.0 ; 1.2 ; 1.4)$ and $\langle n\rangle \approx 0.9$. The Fermi surface topology changes at $J / t=1.2$ (second from right). (Bottom) Fermi surfaces for different dopings across the phase transition between the strong-coupling SDW and the paramagnetic state at $J / t=1.8$ for (from left to right) $\langle n\rangle=(0.98 ; 0.93 ; 0.85)$. The magnetic order vanishes in the middle panel.

of the conduction electrons (top), the spin-spin correlation between localized spins and conduction electrons (middle), and the double occupancy of the conduction electrons (bottom). We only show the expectation values corresponding

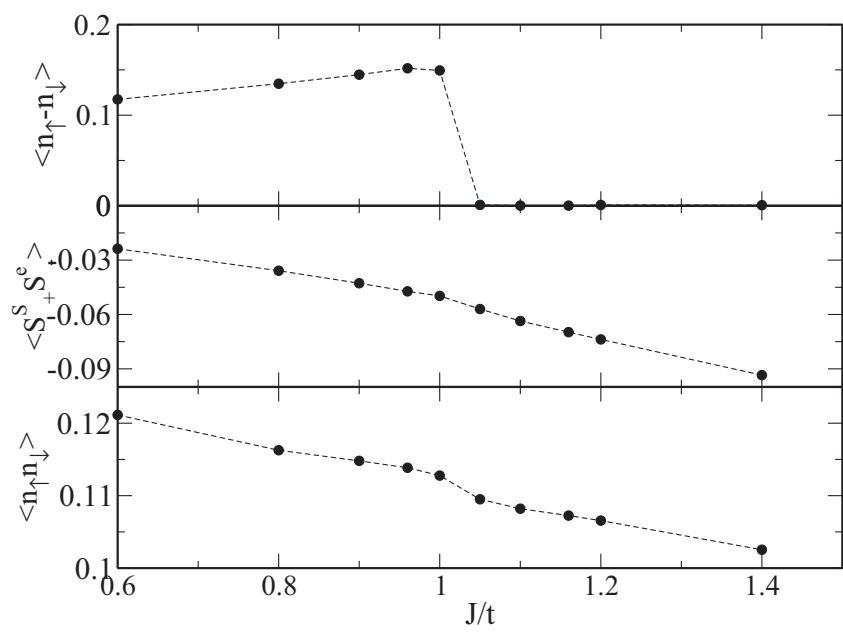

FIG. 7. First-order phase transition between the SDW phase and the paramagnetic phase at weak coupling and $\langle n\rangle \approx 0.8$. The spin polarization as well as the charge density order vanish abruptly at $J / t \approx 1$. The figure shows the electron polarization (top), the spinspin-correlation, $\left\langle S_{+}^{c} S_{-}^{S}\right\rangle$, between conduction electrons and localized moments (middle), and the double occupancy of the conduction electrons (bottom). to the maximally polarized regions of the SDW. As can be clearly seen, the electron polarization vanishes abruptly around $J / t=1$, which signifies a first-order transition between the SDW phase and the paramagnetic phase. We note that within the SDW phase also the electrons in the low-electron-density regions are polarized within this parameter region and that also this polarization jumps abruptly to zero at the phase transition. Exactly at this interaction strength, the Fermi surface changes from small to large. We also observe small discontinuities in the spin-spin correlation and the double occupancy at the phase transition. However, both discontinuities are not very significant. We furthermore note that although the Fermi surface becomes small, the spin-spin correlation does not vanish at weak coupling.

The transition at strong coupling between the SDW phase and the paramagnetic phase looks completely different. We show the polarization of the conduction electrons (top), the spin-spin correlation between localized spins and conduction electrons (middle), and the double occupancy of the conduction electrons (bottom) for conduction-band fillings across the phase transition at $J / t=1.8$ in Fig. 8. Contrary to the phase transition at weak coupling, the electron polarization behaves smoothly upon doping at strong coupling. We observe the well-known second-order phase transition between the SDW phase and the paramagnetic phase. Also the spin-spin correlation and the double occupancy seem to be smooth across the phase transition, although there might be small kinks.

Finally, let us take a look at expectation values across the transition of the Fermi surface inside the SDW phase. 


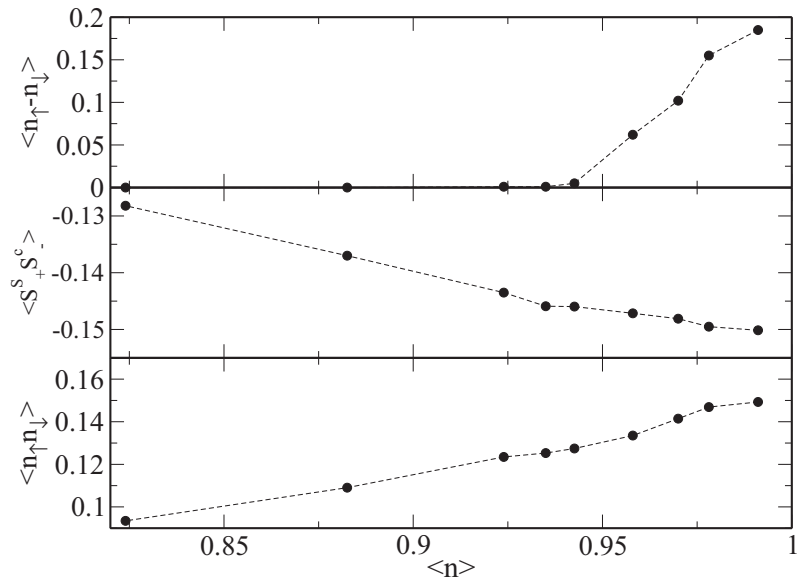

FIG. 8. Second-order phase transition at $J / t=1.8$ for different conduction-band fillings. The figure shows the same quantities as in Fig. 7.

Figure 9 shows the same quantities as shown in Figs. 7 and 8 , but for $\langle n\rangle \approx 0.9$ and different interaction strengths. We have marked the coupling strengths, at which the Fermi surface changes from small to large. At these interaction strengths, we do not observe any signs of a transition within the shown expectation values. All quantities including polarization as well as spin-spin correlations behave smoothly without any kink. Only for large interaction strength, $J / t \approx 1.6$, we observe the second-order phase transition between the SDW phase with large Fermi surface and the paramagnetic phase. Because these results are for the doped system, this transition occurs already for $J / t \approx 1.6$ compared to $J / t \approx 2.2$ at half-filling.

Summarizing our results for the static expectation values, we find a first-order phase transition between the SDW phase with small Fermi surface at weak coupling and the paramagnetic phase, and a second-order phase transition between the SDW phase with large Fermi surface at strong coupling and the paramagnetic phase. The change between these two transitions seems to coincide with the change in

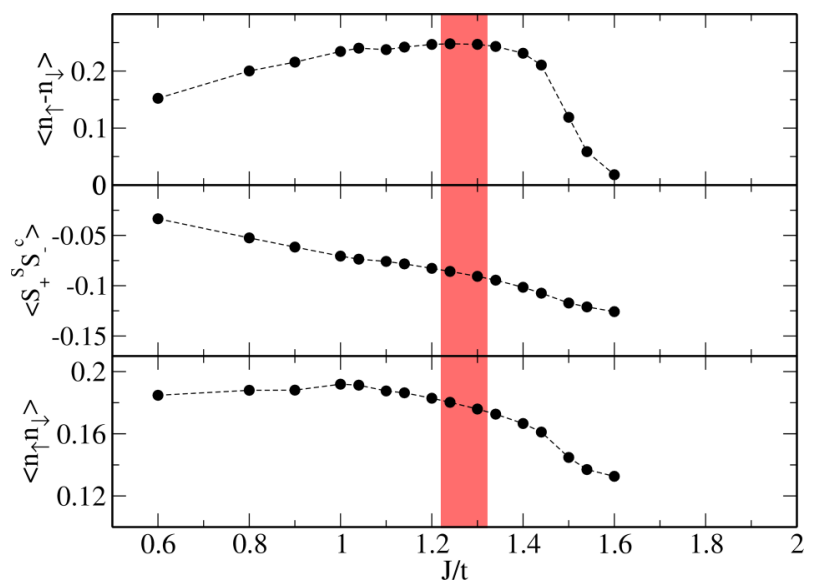

FIG. 9. (Color online) The same quantities as in Figs. 7 and 8 across the Lifshitz transition within the SDW phase. The shaded area corresponds to the interaction strength at which the Fermi surface changes from small to large. the Fermi-surface topology occurring inside the SDW phase. However, we do not observe any sign in the static expectation values, although the Fermi surface changes inside the SDW phase.

\section{CONCLUSIONS}

We have analyzed the phase diagram of the Kondo lattice model close to half-filling by RDMFT. We have demonstrated the existence of incommensurate SDW states which coexist with CDWs in the Kondo lattice model for a wide range of parameters. Although the existence of SDW states can be expected away from half-filling, most of the previous calculations have neglected this possibility.

Remarkably, we have found that within this metallic SDW phase the Fermi surface changes from small at weak coupling to large at strong coupling. This Lifshitz transition, which does not seem to affect static quantities such as polarization and spin-spin correlations, is accompanied by a change in the order of the phase transition between the SDW phase and the heavy fermion paramagnetic phase. While we have found a first-order transition between the weak-coupling small Fermisurface SDW state and the large Fermi-surface paramagnetic state, we have found a continuous transition between the large Fermi-surface SDW at strong coupling and the large Fermisurface paramagnetic state.

We want to note that a similar transition between a large Fermi-surface and a small Fermi-surface antiferromagnetic state has been found in variational Monte Carlo (VMC) calculations [72-75], although in these calculations incommensurate SDW states have not been analyzed. These VMC calculations have also shown that the order of the phase transition between the paramagnetic state and the antiferromagnetic state changes from first order at weak coupling to second order at strong coupling. Furthermore, these VMC calculations have confirmed the Lifshitz transition inside the antiferromagnetic phase. Thus, our RDMFT calculations, which take incommensurate SDW states into account, agree with these previous VMC calculations. Antiferromagnetic states with small and large Fermi surface have also been found in a different DMFT study [53] and dynamical cluster approximation (DCA) studies [76,77] using the Néel state away from half-filling instead of SDWs. Finally, we want to note that a similar Lifshitz transition has been observed in the ferromagnetic phase of the Kondo lattice model [54]. Thus a change in the Fermi-surface topology when increasing the interaction strength seems to be a general property of the Kondo lattice model and not specific to the SDW phase close to half-filling.

Comparing our results to the global phase diagram for heavy fermions, we observe two important differences in our results. Although we find an SDW phase with small Fermi surface and the other with large Fermi surface, the phase transition between the SDW phase with small Fermi surface and the paramagnetic state is of first order, while it is of second order in the global phase diagram. Second, the spin-spin correlations do not vanish in the SDW phase with small Fermi surface. Thus we do not observe a local quantum critical point in the Kondo lattice model within the DMFT approximation, which is supposed to be between the small Fermi-surface 
SDW phase and the paramagnetic phase. This phase transition turns out to be first order in our calculations. We only find the previously known continuous quantum phase transition at strong coupling between the large Fermi-surface SDW and the paramagnetic phase. However, we cannot rule out that a local quantum critical point might occur in the frustrated KLM, or including long range-interactions, or by taking into account spatial fluctuations, which are not included in this DMFT study. By including, for example, an intersite exchange, the strength of the RKKY interaction will be increased, while the Kondo effect is not strongly influenced. One can thus expect that the antiferromagnetic phase, and especially the small Fermi-surface antiferromagnetic phase, extends to larger interactions strengths. Thus it might be possible in that case that the Lifshitz transition merges with the continuous quantum phase transition.

\section{ACKNOWLEDGMENTS}

RP thanks for the support through the FPR program of RIKEN. N.K. is supported through KAKENHI Grant Nos. 25400366 and 15H05855. Computer calculations have been done at the RICC supercomputer at RIKEN and the supercomputer of the Institute of Solid State Physics in Japan.
[1] Q. Si, S. Rabello, K. Ingersent, and J. L. Smith, Nature (London) 413, 804 (2001).

[2] Q. Si, Physica B: Condens. Matter 378-380, 23 (2006).

[3] H. v. Löhneysen, A. Rosch, M. Vojta, and P. Wölfle, Rev. Mod. Phys. 79, 1015 (2007).

[4] P. Coleman, in Handbook of Magnetism and Advanced Magnetic Materials (Wiley, New York, 2007), p. 95.

[5] P. Coleman and A. J. Schofield, Nature (London) 433, 226 (2005).

[6] P. Gegenwart, Q. Si, and F. Steglich, Nat. Phys. 4, 186 (2008).

[7] W. Knafo, S. Raymond, P. Lejay, and J. Flouquet, Nat. Phys. 5, 753 (2009)

[8] Q. Si, Physica Status Solidi (b) 247, 476 (2010).

[9] Q. Si and F. Steglich, Science 329, 1161 (2010).

[10] Q. Si and S. Paschen, Physica Status Solidi (b) 250, 425 (2013).

[11] S. Paschen and Julio Larrea J., J. Phys. Soc. Jpn. 83, 061004 (2014).

[12] J. A. Hertz, Phys. Rev. B 14, 1165 (1976).

[13] A. J. Millis, Phys. Rev. B 48, 7183 (1993).

[14] T. Moriya and T. Takimoto, J. Phys. Soc. Jpn. 64, 960 (1995).

[15] P. Coleman, C. Ppin, Q. Si, and R. Ramazashvili, J. Phys.: Condens. Matter 13, R723 (2001).

[16] P. Gegenwart, J. Custers, C. Geibel, K. Neumaier, T. Tayama, K. Tenya, O. Trovarelli, and F. Steglich, Phys. Rev. Lett. 89, 056402 (2002).

[17] J. Custers, P. Gegenwart, H. Wilhelm, K. Neumaier, Y. Tokiwa, O. Trovarelli, C. Geibel, F. Steglich, C. Pepin, and P. Coleman, Nature (London) 424, 524 (2003).

[18] S. Paschen, T. Luhmann, S. Wirth, P. Gegenwart, O. Trovarelli, C. Geibel, F. Steglich, P. Coleman, and Q. Si, Nature (London) 432, 881 (2004)

[19] P. Gegenwart, T. Westerkamp, C. Krellner, Y. Tokiwa, S. Paschen, C. Geibel, F. Steglich, E. Abrahams, and Q. Si, Science 315, 969 (2007)

[20] S. Hartmann, N. Oeschler, C. Krellner, C. Geibel, S. Paschen, and F. Steglich, Phys. Rev. Lett. 104, 096401 (2010).

[21] S. Friedemann, N. Oeschler, S. Wirth, C. Krellner, C. Geibel, F. Steglich, S. Paschen, S. Kirchner, and Q. Si, Proc. Natl. Acad. Sci. USA 107, 14547 (2010).

[22] T. Senthil, M. Vojta, and S. Sachdev, Phys. Rev. B 69, 035111 (2004).

[23] S. J. Yamamoto and Q. Si, Phys. Rev. Lett. 99, 016401 (2007).

[24] M. Vojta, Phys. Rev. B 78, 125109 (2008).

[25] S. Yamamoto and Q. Si, Physica B: Condens. Matter 403, 1414 (2008).
[26] S. Yamamoto and Q. Si, J. Low Temp. Phys. 161, 233 (2010).

[27] Q. Si, J. H. Pixley, E. Nica, S. J. Yamamoto, P. Goswami, R. Yu, and S. Kirchner, J. Phys. Soc. Jpn. 83, 061005 (2014).

[28] S. Doniach, Physica B+C 91, 231 (1977).

[29] C. Lacroix and M. Cyrot, Phys. Rev. B 20, 1969 (1979).

[30] P. Fazekas and E. Müller-Hartmann, Z. Phys. B Condens. Matter 85, 285 (1991).

[31] H. Tsunetsugu, Phys. Rev. B 55, 3042 (1997).

[32] F. F. Assaad, Phys. Rev. Lett. 83, 796 (1999).

[33] W. Metzner and D. Vollhardt, Phys. Rev. Lett. 62, 324 (1989).

[34] T. Pruschke, M. Jarrell, and J. Freericks, Adv. Phys. 44, 187 (1995).

[35] A. Georges, G. Kotliar, W. Krauth, and M. J. Rozenberg, Rev. Mod. Phys. 68, 13 (1996).

[36] S. Burdin, A. Georges, and D. R. Grempel, Phys. Rev. Lett. 85, 1048 (2000).

[37] J.-X. Zhu, D. R. Grempel, and Q. Si, Phys. Rev. Lett. 91, 156404 (2003).

[38] K. S. D. Beach, P. A. Lee, and P. Monthoux, Phys. Rev. Lett. 92, 026401 (2004).

[39] T. Ohashi, S. ichiro Suga, and N. Kawakami, J. Phys.: Condens. Matter 17, 4547 (2005).

[40] R. Peters and T. Pruschke, Phys. Rev. B 76, 245101 (2007).

[41] K. S. D. Beach and F. F. Assaad, Phys. Rev. B 77, 205123 (2008).

[42] J. Otsuki, H. Kusunose, and Y. Kuramoto, J. Phys. Soc. Jpn. 78, 034719 (2009).

[43] J. Otsuki, H. Kusunose, and Y. Kuramoto, Phys. Rev. Lett. 102, 017202 (2009).

[44] S. Hoshino, J. Otsuki, and Y. Kuramoto, Phys. Rev. B 81, 113108 (2010).

[45] O. Bodensiek, R. itko, R. Peters, and T. Pruschke, J. Phys.: Condens. Matter 23, 094212 (2011).

[46] A. Benlagra, T. Pruschke, and M. Vojta, Phys. Rev. B 84, 195141 (2011).

[47] R. Peters, N. Kawakami, and T. Pruschke, J. Phys.: Conf. Ser. 320, 012057 (2011).

[48] R. Peters, N. Kawakami, and T. Pruschke, Phys. Rev. Lett. 108, 086402 (2012).

[49] M. Bercx and F. F. Assaad, Phys. Rev. B 86, 075108 (2012).

[50] O. Bodensiek, R. Žitko, M. Vojta, M. Jarrell, and T. Pruschke, Phys. Rev. Lett. 110, 146406 (2013).

[51] R. Peters, S. Hoshino, N. Kawakami, J. Otsuki, and Y. Kuramoto, Phys. Rev. B 87, 165133 (2013).

[52] R. Peters, Y. Tada, and N. Kawakami, Phys. Rev. B 88, 155134 (2013). 
[53] S. Hoshino and Y. Kuramoto, Phys. Rev. Lett. 111, 026401 (2013).

[54] D. Golež and R. Žitko, Phys. Rev. B 88, 054431 (2013).

[55] Y. Kuramoto and S. Hoshino, J. Phys. Soc. Jpn. 83, 061007 (2014).

[56] T. Kikuchi, S. Hoshino, and Y. Kuramoto, J. Phys. Soc. Jpn. 83, 114706 (2014).

[57] Žiga. Osolin, T. Pruschke, and R. Žitko, Phys. Rev. B 91, 075105 (2015).

[58] J. Otsuki, Phys. Rev. Lett. 115, 036404 (2015).

[59] L. P. Regnault, W. A. C. Erkelens, J. Rossat-Mignod, P. Lejay, and J. Flouquet, Phys. Rev. B 38, 4481 (1988).

[60] O. Stockert, E. Faulhaber, G. Zwicknagl, N. Stüßer, H. S. Jeevan, M. Deppe, R. Borth, R. Küchler, M. Loewenhaupt, C. Geibel et al., Phys. Rev. Lett. 92, 136401 (2004).

[61] W. Bao, P. G. Pagliuso, J. L. Sarrao, J. D. Thompson, Z. Fisk, J. W. Lynn, and R. W. Erwin, Phys. Rev. B 62, R14621 (2000).

[62] M. Hamada and H. Shimahara, Phys. Rev. B 51, 3027 (1995).

[63] K. G. Wilson, Rev. Mod. Phys. 47, 773 (1975).

[64] R. Bulla, T. A. Costi, and T. Pruschke, Rev. Mod. Phys. 80, 395 (2008).

[65] R. Peters, T. Pruschke, and F. B. Anders, Phys. Rev. B 74, 245114 (2006).
[66] A. Weichselbaum and J. von Delft, Phys. Rev. Lett. 99, 076402 (2007).

[67] R. Peters and N. Kawakami, Phys. Rev. B 89, 155134 (2014).

[68] P. Abbamonte, A. Rusydi, S. Smadici, G. D. Gu, G. A. Sawatzky, and D. L. Feng, Nat. Phys. 1, 155 (2005).

[69] K. Ishida, K. Okamoto, Y. Kawasaki, Y. Kitaoka, O. Trovarelli, C. Geibel, and F. Steglich, Phys. Rev. Lett. 89, 107202 (2002).

[70] S. Lausberg, A. Hannaske, A. Steppke, L. Steinke, T. Gruner, L. Pedrero, C. Krellner, C. Klingner, M. Brando, C. Geibel et al., Phys. Rev. Lett. 110, 256402 (2013).

[71] J. Bauer and A. C. Hewson, Eur. Phys. J. B 57, 235 (2007).

[72] H. Watanabe and M. Ogata, Phys. Rev. Lett. 99, 136401 (2007).

[73] H. Watanabe and M. Ogata, Physica B: Condens. Matter 403, 1390 (2008).

[74] N. Lanatà, P. Barone, and M. Fabrizio, Phys. Rev. B 78, 155127 (2008).

[75] M. Z. Asadzadeh, F. Becca, and M. Fabrizio, Phys. Rev. B 87, 205144 (2013)

[76] L. C. Martin and F. F. Assaad, Phys. Rev. Lett. 101, 066404 (2008).

[77] L. C. Martin, M. Bercx, and F. F. Assaad, Phys. Rev. B 82, 245105 (2010) 